\title{
Upregulated miR-665 expression independently predicts poor prognosis of lung cancer and facilitates tumor cell proliferation, migration and invasion
}

\author{
JINBING XIA ${ }^{1 *}$, DENGPING LI $^{2 *}$, XIAOLIANG ZHU ${ }^{3}$, WENYING XIA ${ }^{4}$, \\ ZHENYONG QI ${ }^{1}$, GUANHUA $\mathrm{LI}^{5}$ and QIAN XU ${ }^{6}$
}

${ }^{1}$ Clinical Laboratory; ${ }^{2}$ Department of CT Magnetic Resonance, Shouguang People's Hospital, Shouguang, Shandong 262700;

${ }^{3}$ Department of Gastrointestinal Surgery, Yidu Central Hospital of Weifang, Qingzhou, Shandong 262500;

${ }^{4}$ Clinical Laboratory, Shouguang Hospital of Traditional Chinese Medicine; ${ }^{5}$ Department of Respiratory Medicine,

Shouguang People's Hospital, Shouguang, Shandong 262700; ${ }^{6}$ Department of Medical Oncology,

Weifang People's Hospital, Weifang, Shandong 261041, P.R. China

Received June 22, 2019; Accepted February 14, 2020

DOI: $10.3892 / 01.2020 .11457$

\begin{abstract}
Non-small cell lung cancer (NSCLC) is one of the leading causes of global cancer-associated mortality. Aberrant microRNAs (miRs) have been reported to be involved in the pathogenesis of various cancer types. The present study aimed to investigate the expression profile and prognostic value of miR-665 in patients with NSCLC, and to analyze its functional role in tumor progression using NSCLC cells. Reverse transcription-quantitative PCR was used to estimate the expression levels of miR-665. Kaplan-Meier survival curves and Cox regression analysis were performed to evaluate the prognostic value of miR-665. The effects of miR-665 on NSCLC cell proliferation, migration and invasion were examined by cell transfection, and the target gene of miR-665 was explored. miR-665 expression was elevated in the tissue and cell samples of NSCLC. This increased miR-665 expression was associated with lymph node metastasis and TNM stage. An independent association between miR-665 and overall survival was identified in patients with NSCLC. When regulating the expression levels of miR-665 in vitro, NSCLC cell proliferation, migration and invasion were enhanced by overexpression of miR-665, but were inhibited by knockdown of miR-665. The luciferase activity results indicated that the protein tyrosine phosphatase receptor type B (PTPRB) was a direct target of miR-665 in NSCLC cells. The present
\end{abstract}

Correspondence to: Dr Qian Xu, Department of Medical Oncology, Weifang People's Hospital, 151 Guangwen Road, Weifang, Shandong 261041, P.R. China

E-mail: qianxujinbu0601@163.com

*Contributed equally

Key words: non-small cell lung cancer, microRNA-665, prognosis, proliferation, migration, invasion study provided evidence for the clinical significance of a decreased expression of miR-665 in the prognosis of NSCLC. Upregulation of miR-665 contributed to tumor cell proliferation, migration and invasion by targeting PTPRB, suggesting the potential of miR-665 as a candidate therapeutic target for NSCLC treatment.

\section{Introduction}

Lung cancer remains one of the most serious global health burdens and represents a dominant cause of cancer-associated mortality (1). Among all cases of lung cancer, non-small cell lung cancer (NSCLC) is the most frequent type, accounting for $\sim 80 \%$ of all cases (2). NSCLC consists of squamous cell carcinoma, adenocarcinoma, adenosquamous cell carcinoma and large cell carcinoma (3). The majority of patients with NSCLC are diagnosed with advanced tumors, and the 5-year survival rates in NSCLC range from $73 \%$ in stage IA disease to $13 \%$ in stage IV disease, worldwide (4). Despite great progress in therapeutic approaches for cancer treatment, such as surgery, chemotherapy, radiotherapy and targeted therapy, the prognosis and outcomes remain poor (5). To improve the management of NSCLC, efforts should be made in the development of novel and efficient strategies for diagnosis, prognosis and treatment.

Several studies have demonstrated that tumor initiation and development involve changes in the expression levels of numerous key molecules (6-8). MicroRNAs (miRNAs/miRs) are a group of these key molecules that have been investigated in various types of cancer $(9,10)$. These small RNAs have no capacity of protein coding, but exert regulatory roles in gene expression at the post-transcriptional level (11). In addition, miRNAs are involved in the regulation of various cell processes, such as cell proliferation, migration, invasion, differentiation, cell cycle and apoptosis $(12,13)$. In cancer research, a large number of miRNAs have been reported to be involved in tumor progression, which means that these functional miRNAs may be considered as potential therapeutic targets for cancer treatment $(14,15)$. Furthermore, the 
clinical significance of miRNAs has attracted increasing attention in the diagnosis and prognosis of human malignancies, including NSCLC (16). miR-665 has been reported to be closely associated with pivotal signaling pathways in lung cancer pathogenesis (17), and miR-665 expression in extracellular vesicles is elevated in NSCLC (18). However, the clinical significance and biological function of miR-665 in NSCLC remain unclear.

To improve the prognosis and treatment of NSCLC, the present study aimed to investigate the expression profile and prognostic value of miR-665 in patients with NSCLC. In addition, cell experiments were carried out to uncover the potential functional role of miR-665 in NSCLC tumor progression. The results of the present study may provide a novel biomarker and a therapeutic target for the treatment of this malignancy.

\section{Materials and methods}

Patients and tissue collection. The present study was approved by the Ethics Committee of Shouguang People's Hospital (Shouguang, China) and written informed consent was obtained from all patients prior to the study start. A total of 128 patients diagnosed with NSCLC were recruited between January 2010 and December 2013 at Shouguang People's Hospital. The inclusion criteria were as follows: i) Patients underwent surgical resection and were pathologically diagnosed with NSCLC at the Shouguang People's Hospital, ii) patients did not receive any preoperative antitumor therapy and iii) patients had complete clinicopathological data and follow-up information. Tumor tissues and adjacent normal tissues were collected from patients following surgical excision, snap frozen in liquid nitrogen and stored at $-80^{\circ} \mathrm{C}$. The demographic and clinicopathological patient characteristics are listed in Table I. A 5-year follow-up survey was performed, and the survival status of patients was recorded.

Cell culture and transfection. The NSCLC A549, H1299 and H522 cell lines, and the human bronchial epithelial 16HBE cell line were obtained from The Cell Bank of Type Culture Collection of the Chinese Academy of Sciences. All cells were cultured in RPMI 1640 medium (Gibco; Thermo Fisher Scientific, Inc.) supplemented with 10\% FBS (Gibco; Thermo Fisher Scientific, Inc.), and $100 \mathrm{IU} / \mathrm{ml}$ penicillin and $100 \mu \mathrm{g} / \mathrm{ml}$ streptomycin (Invitrogen; Thermo Fisher Scientific, Inc.) at $37^{\circ} \mathrm{C}$ in a humidified atmosphere containing $5 \% \mathrm{CO}_{2}$.

To regulate miR-665 expression in vitro, cell transfection was performed using Lipofectamine ${ }^{\circledR} 3000$ (Invitrogen; Thermo Fisher Scientific, Inc.) according to the manufacturer's protocol. The synthesized miR-665 mimic and inhibitor $(50 \mathrm{nM})$ were used to upregulate and downregulate the expression levels of miR-665 in tumor cells. In addition, a negative control sequence (miR-NC, $50 \mathrm{nM}$ ) transfected in the tumor cells was used as a control, and cells transfected with the transfection reagent only were set as the mock group. All vectors were synthesized by Shanghai GenePharma Co., Ltd. with the following sequences: miR-665 mimic, 5'-ACCAGG AGGCUGAGGCCCCU-3'; miR-665 inhibitor, 5'-AGGGGC CUCAGCCUCCUGGU-3'; and miR-NC, 5'-UUCUCCGAA CGUGUCACGU-3'. Subsequent experiments were performed $48 \mathrm{~h}$ post-transfection.
RNA extraction and reverse transcription $(R T)$. Total RNA from tissues and cells was extracted using TRIzol reagent (Invitrogen; Thermo Fisher Scientific, Inc.) according to the manufacturer's protocol. The purity and concentration of the RNA were measured using a NanoDrop 2000 (Thermo Fisher Scientific, Inc.). Total RNA was reversed transcribed into cDNA using the PrimeScript RT reagent kit (Takara Bio, Inc.), with the following reaction conditions: $42^{\circ} \mathrm{C}$ for $30 \mathrm{~min}$ and $85^{\circ} \mathrm{C}$ for $5 \mathrm{sec}$.

$R T$-quantitative (q)PCR. The expression levels of miR-665 were measured using RT-qPCR, which was carried out using a SYBR Green I Master Mix kit (Invitrogen; Thermo Fisher Scientific, Inc.) and the 7300 Real-Time PCR system (Applied Biosystems; Thermo Fisher Scientific, Inc.). The following primer sequences were used for qPCR: miR-665 forward, 5'-GCCGAGACCAGGAGGCTGA-3' and reverse, 5'-CTC AACTGGTGTCGTGGA-3'; and U6 forward, 5'-CTCGCT TCGGCAGCACA-3' and reverse, 5'-AACGCTTCACGAATT TGCGT-3'. The following thermocycling conditions were used for qPCR: Initial denaturation at $95^{\circ} \mathrm{C}$ for $10 \mathrm{~min}$; 40 cycles of $95^{\circ} \mathrm{C}$ for $30 \mathrm{sec}, 60^{\circ} \mathrm{C}$ for $15 \mathrm{sec}$ and $72^{\circ} \mathrm{C}$ for $15 \mathrm{sec}$; and a final extension at $72^{\circ} \mathrm{C}$ for $10 \mathrm{~min}$. Relative miR-665 expression was calculated using the $2^{-\Delta \Delta \mathrm{Cq}}$ method (19) and normalized to U6.

Cell proliferation assay. To explore the effect of miR-665 on tumor cell proliferation, the MTT assay was performed to estimate NSCLC cell proliferation after $48 \mathrm{~h}$ of transfection. Cells at a density of $4 \times 10^{5}$ cells/well were seeded into 48 -well plates and cultured at $37^{\circ} \mathrm{C}$ for $72 \mathrm{~h}$. The MTT solution $(5 \mathrm{mg} / \mathrm{ml})$ was added into the wells at $0,24,48$ and $72 \mathrm{~h}$, followed by $4 \mathrm{~h}$ of incubation. Following MTT incubation, the purple formazan crystals were dissolved in DMSO for $1 \mathrm{~h}$ and cell proliferation was subsequently analyzed at a wavelength of $570 \mathrm{~nm}$, using a microplate reader (Thermo Fisher Scientific, Inc.).

Cell migration and invasion analysis. Transwell chambers (Corning Inc.) were used to evaluate the abilities of cell migration and invasion. After $48 \mathrm{~h}$ post-transfection, cells transfected with vectors or only transfection reagent were plated in the upper chambers in serum-free RPMI 1640 medium at a density of $5 \times 10^{4}$ cells/well, while the lower chambers were filled with RPMI 1640 medium supplemented with $10 \%$ FBS. The chambers were kept in a humidified atmosphere containing $5 \% \mathrm{CO}_{2}$ at $37^{\circ} \mathrm{C}$ for $48 \mathrm{~h}$. For the invasion assay, the Transwell membranes were pre-coated with Matrigel (Corning Inc.) at $37^{\circ} \mathrm{C}$ for $1 \mathrm{~h}$. Following incubation, cells in the upper chambers were removed using cotton swabs, while migratory cells in the lower chambers were incubated with $0.1 \%$ crystal violet (Sigma-Aldrich; Merck $\mathrm{KGaA}$ ) for $10 \mathrm{~min}$ at room temperature. Stained cells were counted in five randomly selected fields using an inverted light microscope (magnification, x200; Olympus Corporation).

Luciferase reporter assay. According to the bioinformatics analysis using TargetScan software (version 7.2; http://www. targetscan.org/vert_72), a potential target gene was predicted for miR-665, known as protein tyrosine phosphatase receptor type B (PTPRB). A luciferase reporter assay was performed to confirm the interaction between miR-665 and PTPRB in H1299 
Table I. Association of miR-665 with clinicopathological features of patients with non-small cell lung cancer.

\begin{tabular}{|c|c|c|c|c|}
\hline \multirow[b]{2}{*}{ Features } & \multirow[b]{2}{*}{ Total no. $(\mathrm{n}=128)$} & \multicolumn{2}{|c|}{ miR-665 expression } & \multirow[b]{2}{*}{ P-value } \\
\hline & & Low $(n=60)$ & $\operatorname{High}(\mathrm{n}=68)$ & \\
\hline Age, years & & & & 0.383 \\
\hline$\leq 60$ & 42 & 22 & 20 & \\
\hline$>60$ & 86 & 38 & 48 & \\
\hline Sex & & & & 0.469 \\
\hline Female & 47 & 24 & 23 & \\
\hline Male & 81 & 36 & 45 & \\
\hline Smoking & & & & 0.872 \\
\hline No & 46 & 22 & 24 & \\
\hline Yes & 82 & 38 & 44 & \\
\hline Tumor size $(\mathrm{cm})$ & & & & 0.773 \\
\hline$\leq 3$ & 58 & 28 & 30 & \\
\hline$>3$ & 70 & 32 & 38 & \\
\hline Differentiation & & & & 0.855 \\
\hline Well/moderate & 80 & 37 & 43 & \\
\hline Poor & 48 & 23 & 25 & \\
\hline Lymph node metastasis & & & & $0.035^{\mathrm{a}}$ \\
\hline No & 62 & 35 & 27 & \\
\hline Yes & 66 & 25 & 41 & \\
\hline TNM stage & & & & $0.009^{\mathrm{a}}$ \\
\hline I-II & 61 & 36 & 25 & \\
\hline III-IV & 67 & 24 & 43 & \\
\hline
\end{tabular}

${ }^{\mathrm{a}} \mathrm{P}<0.05$. miR-665, miRNA-665.

cells. The wild-type (WT) and mutant (MT) 3'-untranslated regions (UTRs) of $P T P R B$ were synthesized and separately cloned into the pmiR-GLO dual-luciferase vector (Shanghai GenePharma Co., Ltd.). After $12 \mathrm{~h}$ of cell inoculation, at a density of $5 \times 10^{4}$ cell/well, the combined vectors were co-transfected into H1299 cells with either miR-665 mimic, miR-665 inhibitor or miR-NC using Lipofectamine ${ }^{\circledR} 3000$ (Invitrogen; Thermo Fisher Scientific, Inc.). Following incubation at $37^{\circ} \mathrm{C}$ for $48 \mathrm{~h}$, cells were collected, and firefly and Renilla luciferase activities were detected using a Dual-Luciferase Reporter assay system (Promega Corporation), according to the manufacturer's protocol. Firefly luciferase activity was normalized to Renilla luciferase activity.

Statistical analysis. Statistical analysis was performed using SPSS software (version 21.0; IBM Corp.) and GraphPad Prism software (version 5.0; GraphPad Software, Inc.) Data are presented as the mean \pm standard deviation and all experiments were performed in triplicate. Differences between groups were analyzed using paired Student's t-test or one-way ANOVA followed by Tukey's post-hoc test. Associations between miR-665 and the clinical features of the patients were assessed using a $\chi^{2}$ test. Survival analysis was performed with the Kaplan-Meier method, and a Cox regression analysis was conducted to confirm the prognostic value of miR-665. $\mathrm{P}<0.05$ was considered to indicate a statistically significant difference.

\section{Results}

miR-665 expression in NSCLC. The present study investigated the expression profile of miR-665 in both NSCLC tissue and cell samples. As shown in Fig. 1A, miR-665 expression was significantly upregulated in the NSCLC tissues compared with in non-cancerous tissues. Furthermore, the expression of miR-665 in patients with different lymph node metastasis (LM) status and TNM stages was compared. The results shown in Fig. 1B indicated that patients with positive LM had significantly higher miR-665 expression than those with negative LM. Furthermore, significantly increased miR-665 expression was observed in patients with advanced TNM stage compared with in patients with TNM stage I-II (Fig. 1C). In addition to the tissue samples, a marked increase in the relative expression of miR-665 was also found in the NSCLC cell lines compared with in the normal cell line (Fig. 1D).

Association between miR-665 and the clinicopathological characteristics of the patients. All demographic and clinical features are summarized in Table I, including age, sex, smoking history, tumor size, differentiation, LM and TNM stage. To explore the association of miR-665 with the clinicopathological data, the mean expression value of miR-665 (0.397) was used to divide the patients into a low miR-665 expression group $(n=60)$ and a high miR-665 expression group 

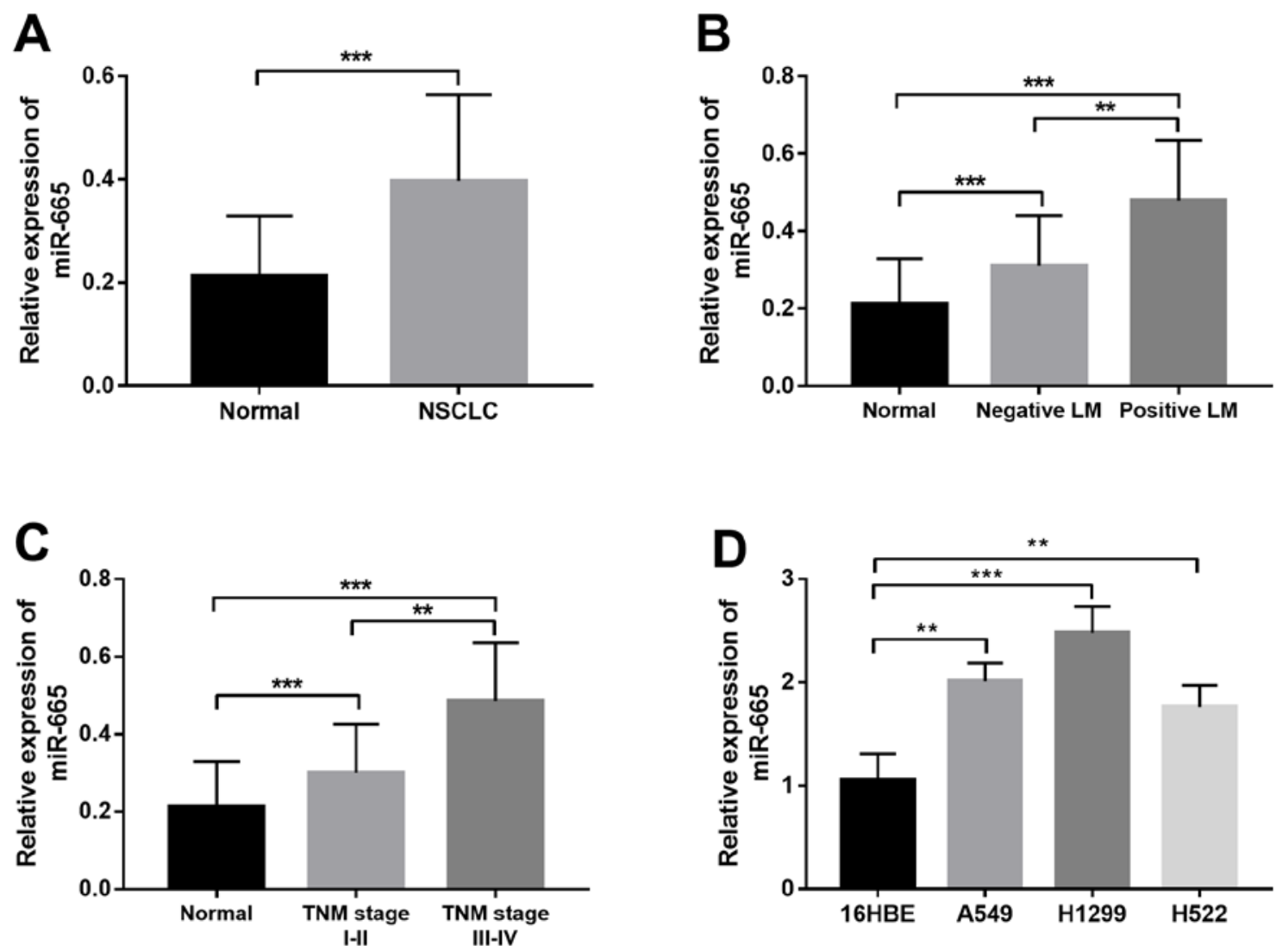

Figure 1. Relative miR-665 expression measured by reverse transcription-quantitative PCR. (A) Relative expression of miR-665 in NSCLC tissues (n=128) was significantly higher compared with that in the normal controls $(n=128)$. (B) Relative expression of miR-665 in patients with positive LM ( $\mathrm{n}=66)$ was significantly higher than in those with negative $\mathrm{LM}(\mathrm{n}=62)$. (C) Relative expression of miR-665 was significantly higher in patients with advanced TNM stage $(\mathrm{n}=67)$ compared with in patients with early TNM stage ( $\mathrm{n}=61)$. (D) Relative miR-665 expression was higher in the three NSCLC cell lines (A549, H1299, H522) compared with normal $16 \mathrm{HBE}$ cells. ${ }^{* *} \mathrm{P}<0.01,{ }^{* * *} \mathrm{P}<0.001$. NSCLC, non-small cell lung cancer; LM, lymph node metastasis; miR-665, miRNA-665.

$(\mathrm{n}=68)$. According to the $\chi^{2}$ test, miR-665 expression was significantly associated with LM and TNM stage. However, no other significant associations between miR-665 expression and the remaining clinical features were observed.

Aberrant expression of miR-665 is independently associated with the overall survival of patients. The present study further evaluated the clinical significance of deregulated miR-665 in the prognosis of NSCLC. The survival curves in Fig. 2A show that patients with low miR-665 expression exhibited improved overall survival compared with those with high miR-665 expression. The association of miR-665 with the overall survival in patients with different TNM stages was further analyzed. This suggested that high miR-665 expression was associated with a shorter survival time in both TNM I-II stage groups (Fig. 2B) and III-IV stage groups (Fig. 2C). The aforementioned data indicated a potential association of miR-665 with the overall survival of patients with NSCLC. Furthermore, the results of the Cox regression analysis shown in Table II revealed that miR-665 expression was independently associated with overall survival, suggesting a prognostic value of miR-665 in patients with NSCLC.

Overexpression of miR-665 contributes to proliferation, migration and invasion of NSCLC cells. The present study aimed to uncover the functional role of miR-665 in NSCLC progression. By transfecting H1299 and A549 cells with a
miR-665 mimic or a miR-665 inhibitor, the expression of miR-665 was upregulated or downregulated, respectively (Fig. 3A and B). The MTT cell proliferation assays revealed that the overexpression of miR-665 in tumor cells led to increased cell proliferation, while the knockdown of miR-665 resulted in suppressed cell proliferation (Fig. 3C and D). In accordance with the proliferation results, the tumor cell migration and invasion were also enhanced by the upregulation of miR- 665 and inhibited by the downregulation of miR-665 (Fig. 4).

PTPRB is a direct target gene of miR-665 in NSCLC cells. Using bioinformatics prediction, a complementary sequence of miR-665 was identified in the 3'-UTR of PTPRB (Fig. 5A). The subsequent luciferase reporter assay results shown in Fig. 5B indicated that the luciferase activity was markedly decreased by miR-665 overexpression, but was promoted by the knockdown of miR-665 in cells with WT 3'-UTR PTPRB. However, no significant changes in the luciferase activity were observed in cells transfected with the MT 3'-UTR PTPRB.

\section{Discussion}

Numerous studies have highlighted the critical roles of the aberrant expression of miRNAs in various diseases, especially in human malignancies (20-22). These functional miRNAs are involved in the occurrence and development of 
A

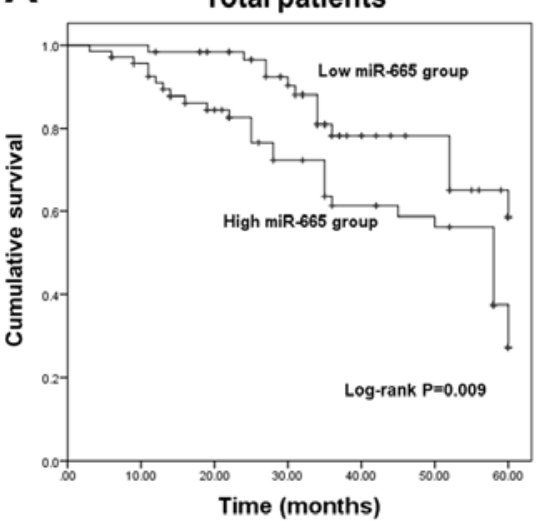

B

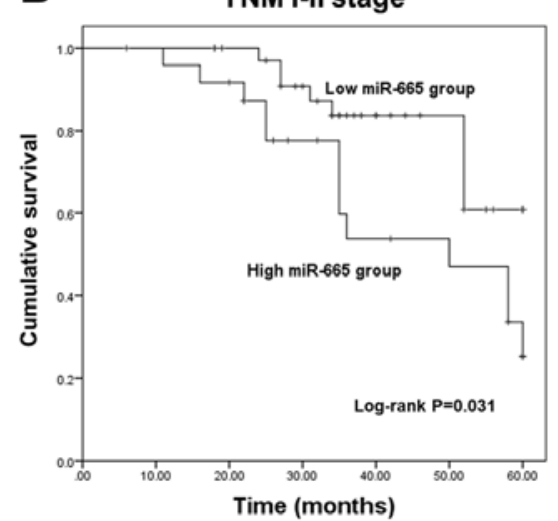

C

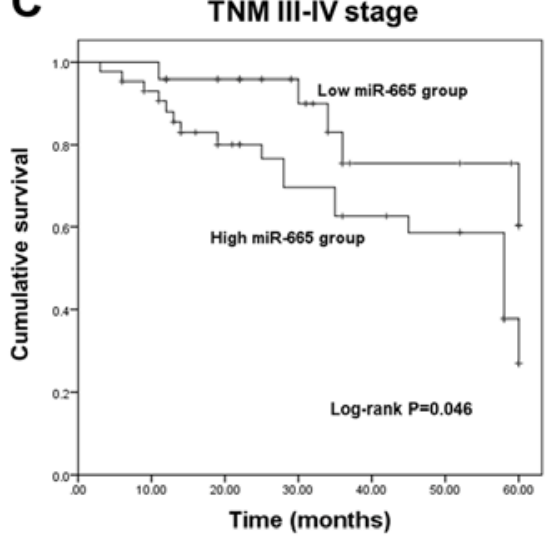

Figure 2. Kaplan-Meier survival curves for patients with non-small cell lung cancer. (A) Patients with low miR-665 levels had longer survival time than those with high miR-665 expression (log-rank $\mathrm{P}=0.009)$. (B) High miR-665 expression was associated with poor overall survival in patients with TNM stages I-II (log-rank P=0.031). (C) High miR-665 expression predicted poor overall survival in patients with advanced TNM stage (log-rank $\mathrm{P}=0.046$ ). miR-665, miRNA-665.

A

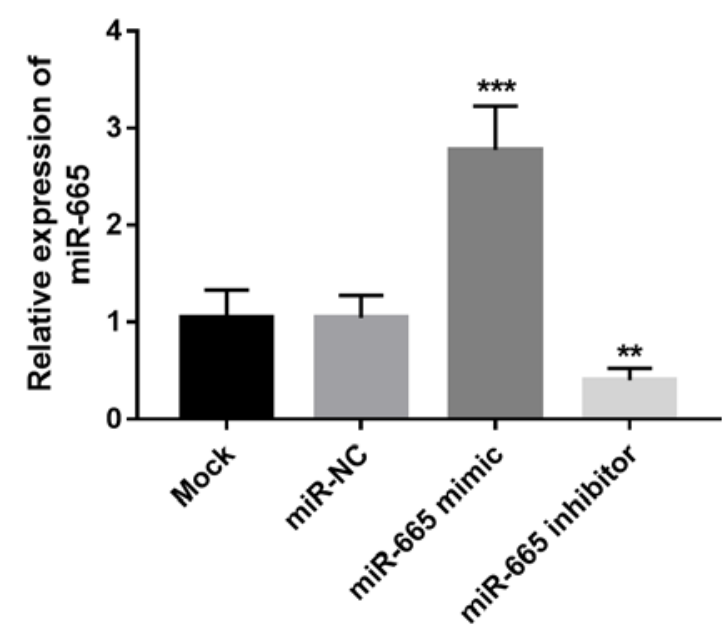

C

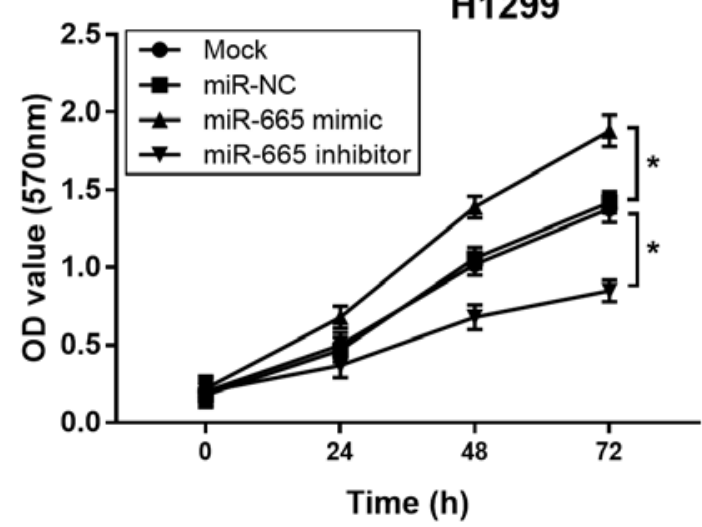

B

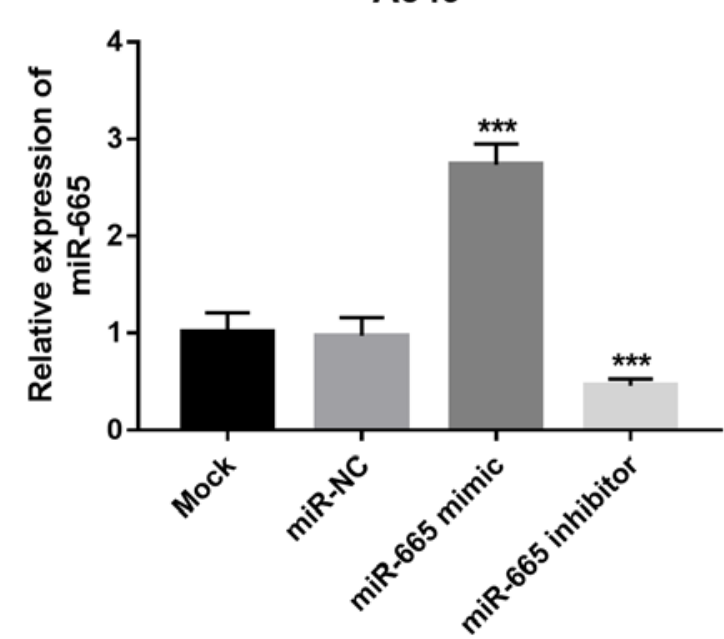

D

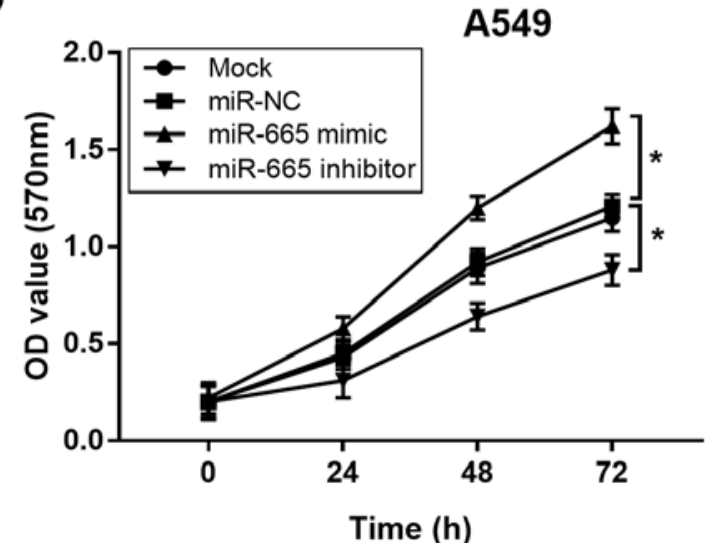

Figure 3. Effects of miR-665 on cell proliferation of H1299 and A549 cells. Expression of miR-665 was significantly increased by the miR-665 mimic, but was suppressed by the miR-665 inhibitor in (A) H1299 and (B) A549 cells. Overexpression of miR-665 in (C) H1299 and (D) A549 cells resulted in increased cell proliferation, whereas the knockdown of miR-665 led to inhibited cell proliferation. ${ }^{*} \mathrm{P}<0.05,{ }^{* *} \mathrm{P}<0.01,{ }^{* * *} \mathrm{P}<0.001$ vs. Mock. NC, negative control; miR-665, miRNA-665; OD, optical density.

tumors, and a number of them have been identified as efficient therapeutic targets for targeted cancer therapy (23). For example, miR-381 has been identified as a tumor suppressor in the progression of pancreatic cancer, demonstrated by the inhibited tumor cell proliferation, migration and invasion, and enhanced cell apoptosis following miR-381 overexpres- 

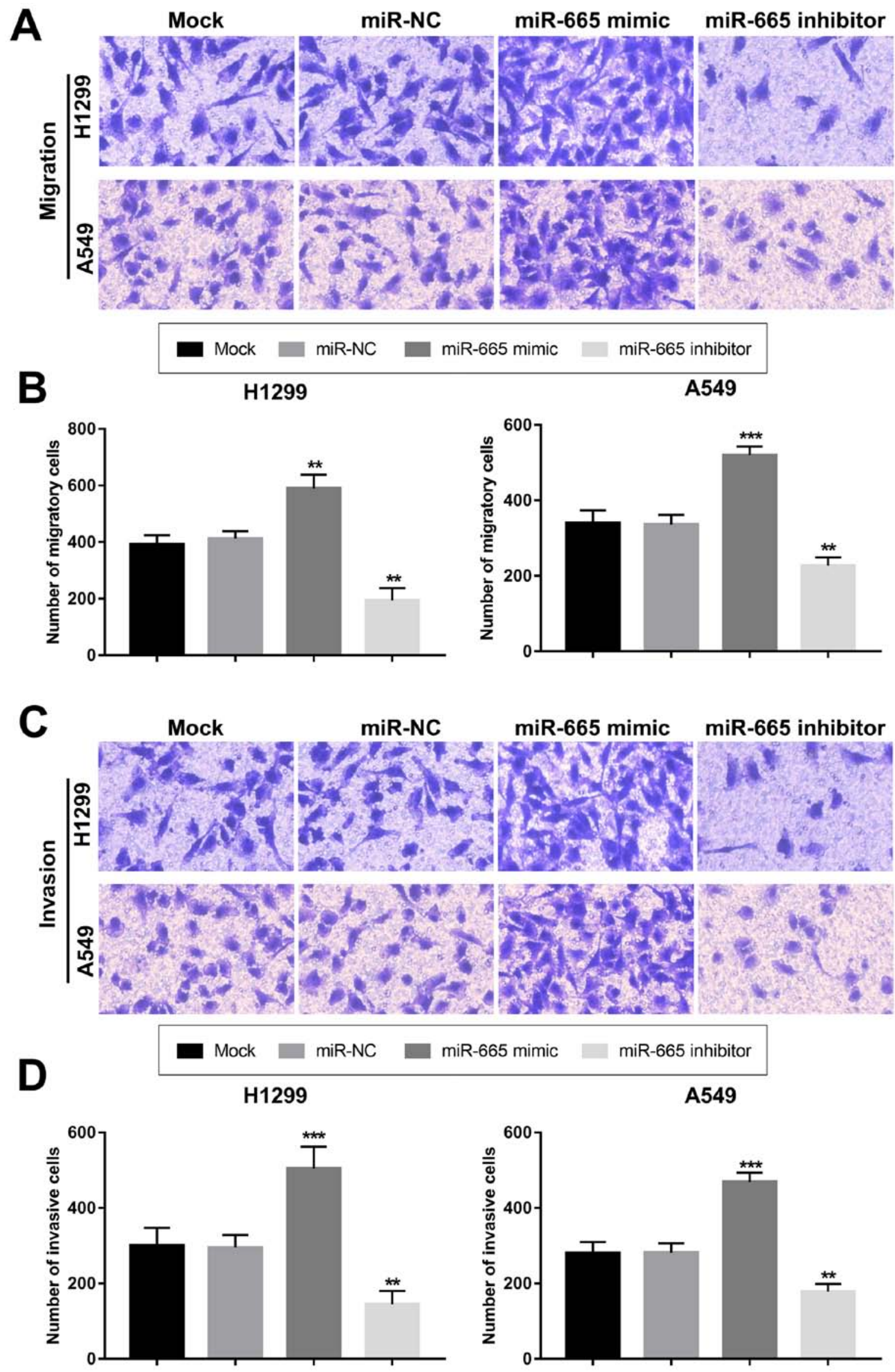

Figure 4. Regulatory role of miR-665 in tumor migration and invasion of non-small cell lung cancer cells. (A) Staining results of Transwell migration assays. (B) Upregulation of miR-665 enhanced migration of both H1299 and A549 cells. (C) Staining results of Transwell invasion assays. (D) Invasion ability of H1299 and A549 cells was facilitated by the overexpression of miR-665, but was suppressed by the inhibition of miR-665. ${ }^{* *} \mathrm{P}<0.01,{ }^{* * *} \mathrm{P}<0.001 \mathrm{vs}$. mock. NC, negative control; miR-665, miRNA-665. 

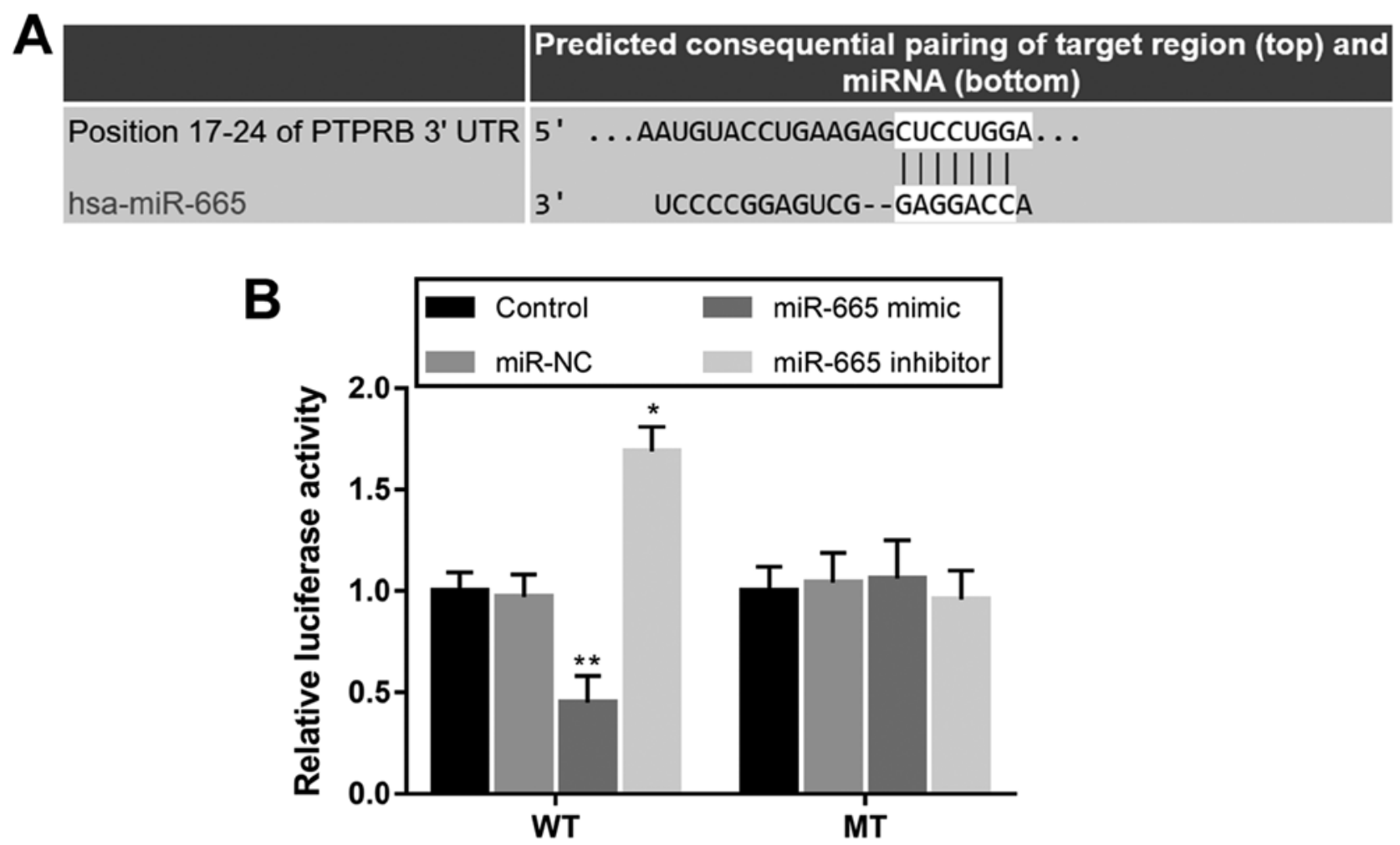

Figure 5. PTPRB serves as a direct target of miR-665 in H1299 cells. (A) Complementary sequence of miR-665 in the 3'-UTR of PTPRB. (B) Overexpression of miR-665 inhibited the luciferase activity, while the knockdown of miR-665 promoted the luciferase activity in cells transfected with the WT 3'-UTR of PTPRB. ${ }^{*} \mathrm{P}<0.05,{ }^{* *} \mathrm{P}<0.01$ vs. control. UTR, untranslated region; PTPRB, protein tyrosine phosphatase receptor type B; NC, negative control; miR-665, miRNA-665; WT, wild-type; MT, mutant.

Table II. Cox regression analysis in patients with non-small cell lung cancer.

\begin{tabular}{lccc}
\hline & \multicolumn{3}{c}{ Multivariate analysis } \\
\cline { 2 - 4 } Variables & HR & $95 \%$ CI & P-value \\
\hline miR-665 & 2.053 & $1.062-3.969$ & $0.032^{\mathrm{a}}$ \\
Age & 0.973 & $0.494-1.914$ & 0.936 \\
Sex & 1.041 & $0.554-1.955$ & 0.901 \\
Smoking & 0.975 & $0.521-1.825$ & 0.938 \\
Tumor size & 1.409 & $0.735-2.701$ & 0.302 \\
Differentiation & 1.005 & $0.535-1.887$ & 0.988 \\
Lymph node metastasis & 0.822 & $0.454-1.488$ & 0.517 \\
TNM stage & 1.816 & $0.932-3.540$ & 0.080 \\
\hline
\end{tabular}

${ }^{\mathrm{a}} \mathrm{P}<0.05$. HR, hazard ratio; miR-665, miRNA-665.

sion (15). Increased expression of miR-1307-3p detected in the hepatocellular carcinoma tissues has been demonstrated to enhance tumor growth and metastasis by regulating disabled homolog 2-interacting protein (24). Additionally, deregulated miR-501 has been demonstrated to be a potential therapeutic target in gastric cancer, and exosomal transfer of miR-501 could regulate the development of this malignancy (25). In addition to the functional roles of miRNAs, their clinical significance has also received increasing attention for the diagnosis and prognosis of various types of cancer, including NSCLC (26). For instance, Sun et al (27) showed that the serum expression levels of miR-30a-5p are downregulated in patients with colorectal carcinoma, and that it could represent a candidate diagnostic and prognostic biomarker. Decreased miR-424-5p expression in hepatocellular carcinoma tissues is independently associated with poor overall survival of the patients, highlighting its value in cancer prognosis (28). In NSCLC, some aberrant miRNAs with dramatic clinical significance have also been identified, such as miR-25 (16) and miR-411 (29).

In the current study, the expression levels of miR-665 were significantly upregulated in the NSCLC tissues and cell lines compared with in the corresponding normal controls, and this increased expression was closely associated with the LM and TNM stage of the patients. Thus, it was hypothesized that miR-665 may serve as an onco-miR and may be involved in the development of NSCLC. Given the dysregulation of miR-665, a survival analysis was performed to evaluate the prognostic significance of miR-665. Patients with high miR-665 levels had shorter survival times compared with those with low miR-665 expression, suggesting that high miR-665 expression was associated with poor overall survival. In addition, by dividing the patients into groups based on TNM stages, it was revealed that high miR-665 expression predicted poor overall survival of patients with both early and advanced TNM stages. Furthermore, the Cox regression analysis demonstrated that miR-665 was an independent prognostic indicator in patients with NSCLC. 
Numerous studies have indicated that miRNAs are involved in the pathogenesis of human cancer by regulating tumor cell processes, such as proliferation, migration and invasion (30-32). Therefore, the present study carried out further cellular experiments to uncover the functional role of miR-665 in NSCLC progression. When regulating miR-665 expression in vitro, the overexpression of miR-665 in NSCLC cells resulted in enhanced cell proliferation, migration and invasion, while the knockdown of miR-665 led to the opposite results. Overall, miR-665 may serve as an enhancer of NSCLC tumorigenesis. Although a promoting effect of miR-665 on tumor cell proliferation was observed, an association between miR-665 expression and tumor size was not detected. This might be due to the small sample size that limited the accuracy of the clinical data. Thus, further investigations with a larger study cohort are necessary. The regulatory effects of miR-665 on tumor cell processes have been previously investigated in other malignancies. In hepatocellular carcinoma cells, miR-665 can promote cell migration, invasion and proliferation by targeting PTPRB (33). In breast cancer, tumor cell migration and invasion are enhanced by the overexpression of miR-665 by promoting the epithelial-mesenchymal transition process (34). By contrast, miR-665 has been demonstrated to suppress cell proliferation and migration in ovarian cancer cells (35). This controversy on the role of miR-665 may be due to the different cancer types. Although the cell experimental data indicated an oncogenic role of miR-665 in NSCLC progression, further studies are required to confirm the biological function of miR-665 by investigating its effects on NSCLC cell cycle distribution and apoptosis, as well as its functional role in tumorigenesis in vivo.

Although the present study provided novel insights on the clinical significance and functional role of miR-665 in NSCLC, the molecular mechanisms underlying the role of miR-665 remain unclear and warrant further investigations. A study by $\mathrm{Hu}$ et al (33) demonstrated that miR-665 exerts promoting effects on hepatocellular carcinoma cell proliferation, migration and invasion by directly targeting PTPRB and through the Hippo signaling pathway. Interestingly, PTPRB has been found to suppress NSCLC cell proliferation and invasion by inhibiting the phosphorylation of the proto-oncogene tyrosine-protein kinase Src (36). In the present study, the binding site of miR-665 was predicted in the 3'-UTR of PTPRB, confirming that $P T P R B$ was a direct target gene of miR-665 in NSCLC. Thus, the miR-665/PTPRB axis may be involved in the regulation of NSCLC progression through the Src and/or Hippo signaling pathways. However, the regulatory effects of miR-665 on PTPRB and the aforementioned signaling pathways in NSCLC were not examined, which represents one of the limitations of the present study and warrants further confirmation to better understand these molecular mechanisms. In addition, other limitations included a relatively small sample size and the lack of cell cycle analysis results in tumor cells with deregulated miR-665 expression. Further studies are required to confirm the role of miR-665 in NSCLC by using a larger sample size and analyzing its regulatory effect on tumor cell biological function.

In conclusion, the results of the present study revealed that upregulated miR-665 expression was associated with the LM and TNM stage of patients with NSCLC, and could be used as a potential prognostic biomarker. NSCLC cell proliferation, migration and invasion were promoted by the overexpression of miR-665, indicating that methods to decrease miR-665 expression could be used as novel therapeutic strategies for NSCLC treatment.

\section{Acknowledgements}

Not applicable.

\section{Funding}

No funding was received.

\section{Availability of data and materials}

The datasets used and/or analyzed during the current study are available from the corresponding author on reasonable request.

\section{Authors' contributions}

JX, DL and QX designed the present study, drafted and revised the initial manuscript for important intellectual content, and acquired and analyzed the data. XZ, WX, ZQ and GL performed the experiments and analyzed the data.

\section{Ethics approval and consent to participate}

The Ethics Committee of Shouguang People's Hospital approved the present study. Written informed consent was obtained from all patients prior to enrollment in the present study.

\section{Patient consent for publication}

Not applicable.

\section{Competing interests}

The authors declare that they have no competing interests.

\section{References}

1. Torre LA, Siegel RL and Jemal A: Lung cancer statistics. Adv Exp Med Biol 893: 1-19, 2016.

2. Akhurst T: Staging of non-small-cell lung cancer. PET Clin 13: $1-10,2018$.

3. Blandin Knight S, Crosbie PA, Balata H, Chudziak J, Hussell T and Dive C: Progress and prospects of early detection in lung cancer. Open Biol 7: pii: 170070, 2017.

4. Woodard GA, Jones KD and Jablons DM: Lung cancer staging and prognosis. Cancer Treat Res 170: 47-75, 2016.

5. Wu DM, Liu T, Deng SH, Han R and Xu Y: SLC39A4 expression is associated with enhanced cell migration, cisplatin resistance, and poor survival in non-small cell lung cancer. Sci Rep 7: 7211, 2017.

6. Xue J, Yang J, Luo M, Cho WC and Liu X: MicroRNA-targeted therapeutics for lung cancer treatment. Expert Opin Drug Discov 12: 141-157, 2017.

7. Huang D, Sun W, Zhou Y, Li P, Chen F, Chen H, Xia D, Xu E, Lai M, Wu Y and Zhang H: Mutations of key driver genes in colorectal cancer progression and metastasis. Cancer Metastasis Rev 37: 173-187, 2018. 
8. Lu J, Zhan Y, Feng J, Luo J and Fan S: MicroRNAs associated with therapy of non-small cell lung cancer. Int J Biol Sci 14: 390-397, 2018.

9. Hwang DW, Kim HY, Li F, Park JY, Kim D, Park JH, Han HS, Byun JW, Lee YS, Jeong JM, et al: In vivo visualization of endogenous miR-21 using hyaluronic acid-coated graphene oxide for targeted cancer therapy. Biomaterials 121: 144-154, 2017.

10. Li Y, Duo Y, Bi J, Zeng X, Mei L, Bao S, He L, Shan A, Zhang Y and $\mathrm{Yu} \mathrm{X}$ : Targeted delivery of anti-miR-155 by functionalized mesoporous silica nanoparticles for colorectal cancer therapy. Int J Nanomedicine 13: 1241-1256, 2018.

11. Liu J, Zhang X, Huang Y, Zhang Q, Zhou J, Zhang X and Wang X: miR-200b and miR-200c co-contribute to the cisplatin sensitivity of ovarian cancer cells by targeting DNA methyltransferases. Oncol Lett 17: 1453-1460, 2019.

12. An JC, Shi HB, Hao WB, Zhu K and Ma B: miR-944 inhibits lung adenocarcinoma tumorigenesis by targeting STAT1 interaction. Oncol Lett 17: 3790-3798, 2019.

13. Dong $\mathrm{M}, \mathrm{Xie} \mathrm{Y}$ and $\mathrm{Xu} \mathrm{Y}$ : miR-7-5p regulates the proliferation and migration of colorectal cancer cells by negatively regulating the expression of Krüppel-like factor 4. Oncol Lett 17: 3241-3246, 2019.

14. Yang F, Chen L and Wang ZJ: MicroRNA-32 inhibits the proliferation, migration and invasion of human colon cancer cell lines by targeting E2F transcription factor 5. Eur Rev Med Pharmacol Sci 23: 4156-4163, 2019.

15. Qiao G, Li J, Wang J, Wang Z and Bian W: miR-381 functions as a tumor suppressor by targeting ETS1 in pancreatic cancer. Int J Mol Med 44: 593-607, 2019.

16. Li J, Yu M, Liu Z and Liu B: Clinical significance of serum miR-25 in non-small-cell lung cancer. Br J Biomed Sci 76: 111-116, 2019.

17. Jin X, Guan Y, Sheng H and Liu Y: Crosstalk in competing endogenous RNA network reveals the complex molecular mechanism underlying lung cancer. Oncotarget 8: 91270-91280, 2017.

18. Li C, Qin F, Hu F, Xu H, Sun G, Han G, Wang T and Guo M: Characterization and selective incorporation of small non-coding RNAs in non-small cell lung cancer extracellular vesicles. Cell Biosci 8: 2, 2018.

19. Livak KJ and Schmittgen TD: Analysis of relative gene expression data using real-time quantitative PCR and the 2(-Delta Delta C(T)) method. Methods 25: 402-408, 2001

20. Zhu C, Huang Q and Zhu H: miR-383 inhibited the cell cycle progression of gastric cancer cells via targeting cyclin E2. DNA Cell Biol 38: 849-856, 2019.

21. Gao W, Zhang Y, Niu M, Bo Y, Li H, Xue X, Lu Y, Zheng X Tang Y, Cui J, et al: Identification of miR-145-5p-centered competing endogenous RNA network in laryngeal squamous cell carcinoma. Proteomics 19: e1900020, 2019.

22. Tao L, Wu YQ and Zhang SP: MiR-21-5p enhances the progression and paclitaxel resistance in drug-resistant breast cancer cell lines by targeting PDCD4. Neoplasma 66: 746-755, 2019.

23. Wang R, Li G, Zhuang G, Sun S and Song Z: Overexpression of microRNA-423-3p indicates poor prognosis and promotes cell proliferation, migration, and invasion of lung cancer. Diagn Pathol 14: 53, 2019.
24. Chen S, Wang L, Yao B, Liu Q and Guoa C: miR-1307-3p promotes tumor growth and metastasis of hepatocellular carcinoma by repressing DAB2 interacting protein. Biomed Pharmacother 117: 109055,2019

25. Liu X, Lu Y, Xu Y, Hou S, Huang J, Wang B, Zhao J, Xia S, Fan S, Yu X, et al: Exosomal transfer of miR-501 confers doxorubicin resistance and tumorigenesis via targeting of BLID in gastric cancer. Cancer Lett 459: 122-134, 2019.

26. Zou JG, Ma LF, Li X, Xu FL, Fei XZ, Liu Q, Bai QL and Dong YL: Circulating microRNA array (miR-182,200b and 205) for the early diagnosis and poor prognosis predictor of non-small cell lung cancer. Eur Rev Med Pharmacol Sci 23: 1108-1115, 2019.

27. Sun Y, Yang B, Lin M, Yu H, Chen $\mathrm{H}$ and Zhang Z: Identification of serum miR-30a-5p as a diagnostic and prognostic biomarker in colorectal cancer. Cancer Biomark 24: 299-305, 2019.

28. Du H, Xu Q, Xiao S, Wu Z, Gong J, Liu C, Ren G and Wu H: MicroRNA-424-5p acts as a potential biomarker and inhibits proliferation and invasion in hepatocellular carcinoma by targeting TRIM29. Life Sci 224: 1-11, 2019.

29. Wang SY, Li Y, Jiang YS and Li RZ: Investigation of serum miR-411 as a diagnosis and prognosis biomarker for non-small cell lung cancer. Eur Rev Med Pharmacol Sci 21: 4092-4097, 2017.

30. Liang Z, Li X, Liu S, Li C, Wang X and Xing J: MiR-141-3p inhibits cell proliferation, migration and invasion by targeting TRAF5 in colorectal cancer. Biochem Biophys Res Commun 514: 699-705, 2019.

31. Chen Z, Gao Y, Gao S, Song D and Feng Y: MiR-135b-5p promotes viability, proliferation, migration and invasion of gastric cancer cells by targeting Krüppel-like factor 4 (KLF4). Arch Med Sci 16: 167-176, 2019.

32. Long X, Shi Y, Ye P, Guo J, Zhou Q and Tang Y: MicroRNA-99a suppresses breast cancer progression by targeting FGFR3. Front Oncol 9: 1473, 2020.

33. Hu Y, Yang C, Yang S, Cheng F, Rao J and Wang X: miR-665 promotes hepatocellular carcinoma cell migration, invasion, and proliferation by decreasing Hippo signaling through targeting PTPRB. Cell Death Dis 9: 954, 2018.

34. Zhao XG, Hu JY, Tang J, Yi W, Zhang MY, Deng R, Mai SJ, Weng NQ, Wang RQ, Liu J, et al: miR-665 expression predicts poor survival and promotes tumor metastasis by targeting NR4A3 in breast cancer. Cell Death Dis 10: 479, 2019.

35. Liu J,Jiang Y, Wan Y,Zhou S, Thapa S and Cheng W: MicroRNA-665 suppresses the growth and migration of ovarian cancer cells by targeting HOXA10. Mol Med Rep 18: 2661-2668, 2018.

36. Qi Y, Dai Y and Gui S: Protein tyrosine phosphatase PTPRB regulates Src phosphorylation and tumour progression in NSCLC. Clin Exp Pharmacol Physiol 43: 1004-1012, 2016.

This work is licensed under a Creative Commons Attribution-NonCommercial-NoDerivatives 4.0 International (CC BY-NC-ND 4.0) License. 